\title{
Hard-Decision Based Reporting Method For Limited Band Control Channel in Cooperative Cognitive Radio Networks
}

\author{
R.A. Mokhtar, ${ }^{*}$, S. Khatun ${ }^{1}$, B.M. Ali $^{2}$ and A. Ramli ${ }^{1}$ \\ ${ }^{I}$ Department of Computer and Communications Systems Engineering, Faculty of Engineering, University Putra Malay- \\ sia, 43400 UPM, Serdang, Selangor, Malaysia \\ ${ }^{2}$ Wireless Communications Centre, MIMOS Berhad, Technology Park Malaysia 57000 Kuala Lumpur, Malaysia
}

\begin{abstract}
In the context of cognitive radio the cooperative spectrum sensing has been proposed to overcome the problem associated with the local sensing for example the hidden node problem due to noise uncertainty, fading, and shadowing. However, sensing results reporting requires perfect channel to avoid the performance degradation. It also requires a large bandwidth under the hypothesis of large number of cognitive user. This paper presents a hard decision auto-correction reporting scheme that able to correct the error in the reported bit directly. This system further minimizes the average number of the reporting bits by allowing only the user with detection information (binary decision 1) to report its result. The sensing performance is investigated and the numerical result shows significant decrease in reporting bits without affecting the sensing performance.
\end{abstract}

\section{INTRODUCTION}

Spectrum sensing is a base aspect of cognitive radio to insure no interference for the primary (licensed user), however the spectrum sensing can be done locally in the node, where it will be susceptible to shadowing and fading that could cause a hidden node problem and will degrade the sensing performance. The cooperative sensing is proposed [1] to overcome the problems associated with the local sensing, different cooperative method is discussed that exploit the multiuser diversity in sensing process [2,3]. Different protocols can be employed in order to report the local sensing result to other secondary user or a common server in centralized or decentralized architecture respectively, the Amplify and forward (AF) in which the relay transmits the signal obtained from the transmitter without any processing achieved full diversity [4]. In [5] the AF is been used in two cognitive user scenario, where each user considered as a relay for other user signal in the next time slot, the scheme show a reduction in detection time and increase agility.

In cooperative sensing architectures, the control channel can be implemented using different methodologies. These include a dedicated band, unlicensed band such as ISM, and underlay ultra wide band (UWB) system [6]. In order to minimize communication overhead, different quantization of the local obtained signal is introduced. It was shown that two or three bits quantization was most appropriate without noticeable loss in the performance [7]. In [8] a hard decision (binary quantization) is proposed for arbitrary large node population. However, the total number of sensing bits transmitted to the central is still very huge.

*Address correspondence to this author at the Department of Computer and Communications Systems Engineering, Faculty of Engineering, University Putra Malaysia, 43400 UPM, Serdang, Selangor, Malaysia;

E-mail: gs15829@mutiara.upm.edu.my
Further to minimize reporting bandwidth a two level quantization method was recently proposed in [9], the method identify the users with a reliable information only to report a binary decision $(0,1)$ to the common server as shown in Fig. (1b), however the method reduce the number of reporting bits but with a degradation in sensing performance Their result show that misdetection probability $P_{m}$ is degraded by the imperfect channel and the false alarm probability $P_{f}$ is bounded by the reporting error probability. This means that spectrum sensing cannot be successfully conducted when the desired $P_{f}$ smaller than the bound $\bar{P}_{f}$. If the channels between cognitive users and the central server are perfect the local decision will send to central server without error, in practice, the reporting channels may experience fading which will deteriorate the performance of the cooperative spectrum sensing. A cluster based method was proposed in [10] where the most favorable user in each cluster is selected to report to central server, the method improved the sensing performance comparing to conventional sensing.

In this paper, we propose a reporting scheme that auto correct the error in the sensing results reported to the central server. By allowing only the users with detection information to report their local detection result to the central Server, the Server will know that whatever signal it receive from a cognitive users it mean a detection of a primary user (local decision 1), if it receive an error signal due to imperfect reporting channel it auto correct it to 1 . The method has two advantages: it reduces the number of the reporting bits and it corrects the error in reporting signal. Further the scheme avoids the need of a decision fusion method at the central server. The sensing and detection of primary users was evaluated that insure the method does not degrade the sens- 
ing performance and the algorithm act as it has a perfect reporting channel.

The rest of this paper is organized as fellow. In section II proposed the auto correction reporting scheme and discusses the system model. Section III analyzes the performance of cooperative sensing for the scheme, and the simulation result is shown. Section IV concludes the paper.

\section{SYSTEM MODEL}

In this proposed model, every cognitive user conduct a local sensing and if a primary user detected, a hard decision ' 1 ' ' is sent to central server, other wise no action is taken, If the server receives a local decision ' 0 ' due to imperfect reporting channel, according to a pre-knowledge, it is able to auto correct the reported error, to make it ' 1 '.

For simplicity energy detection based sensing is assumed for the local sensing method where the output of the integrator, $Q$, is compared with a threshold, $\lambda$, to decide the presence of a primary user.

If $Q$ exceed the threshold, a reporting decision, $R$, is taken and binary decision ' 1 ' is sent to central server otherwise "no report" decision, $R^{\prime}$, is taken. This is given by:

$$
R=\left\{\begin{array}{lll}
0 & Q<\lambda & R^{\prime} \\
1 & Q>\lambda & R
\end{array}\right.
$$

The considered system model of our interest is shown in Fig. (1a).

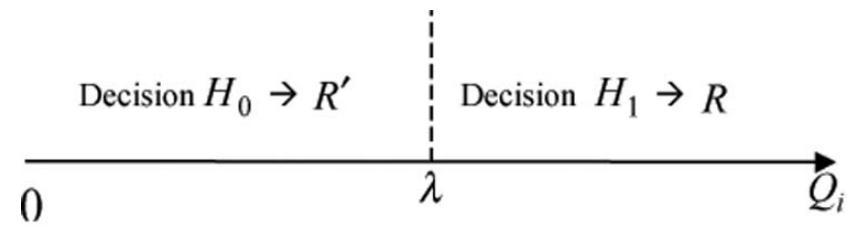

(a)

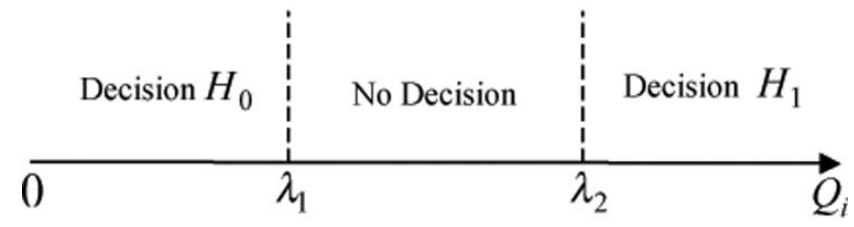

(b)

Fig. (1). (a) Auto_correction reporting method with one threshold for the ith user, (b) Censoring detection method with bi-thresholds for the ith user.

Following the work of [11], where the white noise and the signal term are modeled as zero mean Gaussian random variable, the decision metric $R$ of the energy detector follows the following distribution:

$$
R \sim\left\{\begin{array}{lll}
X_{2 m}^{2} & H_{0}, & R^{\prime} \\
X_{2 m}^{2}(2 \gamma) & H_{1}, & R
\end{array}\right.
$$

where $m$ is the time bandwidth product. $X_{2 m}^{2}$ and $X_{2 m}^{2}(2 \gamma)$ represents the central and non-central chi-square distribution with $2 m$ degree of freedom respectively and $2 \gamma$ non centrality parameter for the later. The SNR $\gamma$ is exponentially distributed with mean value $\bar{\gamma}$ for Rayleigh fading channel.

Assume that the receiver receives $K$ (where $K=0,1, \ldots \ldots, N$ ) out of $N$ local decision 1 reported form the cognitive users. The final decision $H$ at the server is done based on $\mathrm{K}$. if the server receives any local decision 1 or 0 a final decision $H=1$ is taken. If no local decision is reported to the server, then a final decision $H=0$ is taken. $H$ is given by

$H= \begin{cases}1 & K \geq 1 \\ 0 & K=0\end{cases}$

Let $K^{\prime}$ denote the normalize average number of reporting bits

$K^{\prime}=\frac{K_{a v g}}{N}$

where $N$ is the maximum number of reporting bits (for maximum $N$ users in the system).

If $R_{K}$ represents that $K$ cognitive users has reported, then the number of users without report is $N-K$ can be represented as $\bar{R}_{N-K}$. This can be modeled as:

$$
\begin{aligned}
& P\left\{R_{K}\right\}=1-P\{Q<\lambda\} \\
& P\left\{R_{N-K}\right\}=P\{Q<\lambda\}
\end{aligned}
$$

Further, let $P_{0}=P\left\{H_{0}\right\}, P_{1}=P\left\{H_{1}\right\}$. Then the average number of reporting bits is:

$$
\begin{aligned}
K_{\text {avg }}= & P_{0} \sum_{K=0}^{N} K\left(\begin{array}{l}
N \\
K
\end{array}\right) P\left\{\bar{R}_{N-K} \mid H_{0}\right\} \\
& +P_{1} \sum_{K=0}^{N} K\left(\begin{array}{l}
N \\
K
\end{array}\right) P\left\{R_{K} \mid H_{1}\right\} \\
K^{\prime}=1- & P_{0} R_{0}^{\prime}-P_{1} R_{1}^{\prime}
\end{aligned}
$$

Where $R_{0}^{\prime}, R_{1}^{\prime}$ represent the probability of 'no report' under hypothesis $H_{0}, H_{1}$ respectively.

$$
R_{0}^{\prime}=P\left\{Q<\lambda \mid H_{0}\right\}, \quad R_{1}^{\prime}=P\left\{Q<\lambda \mid H_{1}\right\}
$$

From equation (8) it can be shown that, the normalized average number of reporting bits $K^{\prime}$ is always smaller than 1.

\section{PERFORMANCE ANALYSIS}

If the channel between the cognitive users and the central server are perfect, and a full reporting is employed, the detection probability, $P_{d}$, the false alarm probability, $P_{f}$, and the misdetection probability, $P_{m}$, are given by [10] as: 


$$
\begin{aligned}
& P_{d}=1-\prod_{k=1}^{N}\left(1-P_{d, k}\right), \\
& P_{f}=1-\prod_{k=1}^{N}\left(1-P_{f, k}\right),
\end{aligned}
$$

and

$$
P_{m}=\prod_{k=1}^{N} P_{m, k}
$$

where $P_{d, k}, P_{f, k}, P_{m, k}$ are the detection probability, the false alarm probability, and the misdetection probability for the $k$ th cognitive user, respectively.

Under Rayleigh fading, $\boldsymbol{\gamma}$, would have an exponential distribution. In this case, the Cumulative Distribution Function (CDF) of collected energy, $Q$, under hypothesis $H_{0}, H_{1}$ is

$$
F(\lambda)=\int_{0}^{\lambda} f\left(Q \mid H_{0}\right) d Q=1-\frac{\Gamma\left(m, \frac{\lambda}{2}\right)}{\Gamma(m)},
$$

and

$$
\begin{aligned}
G(\lambda) & =\int_{0}^{\lambda} f\left(Q \mid H_{1}\right) d Q \\
& =1-e^{-\frac{\lambda}{2}} \sum_{k=0}^{m} \frac{1}{k !}\left(\frac{\lambda}{2}\right)^{k}+\left(\frac{1+\bar{\gamma}}{\bar{\gamma}}\right)^{m-1} \\
& \times\left(e^{-\frac{\lambda}{2(1+\bar{\gamma})}}-e^{-\frac{\lambda}{2}} \sum_{k=0}^{m-2} \frac{1}{k !}\left(\frac{\lambda \bar{\gamma}}{2(1+\bar{\gamma})}\right)^{k}\right)
\end{aligned}
$$

Then $R_{0}^{\prime}, R_{1}^{\prime}$ can be written as:

$R_{0}^{\prime}=F(\lambda), \quad R_{1}^{\prime}=G(\lambda)$

Where $\Gamma(.,),. \Gamma($.)are incomplete and complete gamma functions respectively [12].

In case $K=0$, no report is sent to the server, that is, no primary user is active in the frequency band. Let $\beta_{0}, \beta_{1}$ denote the probability of 'no report' under hypothesis $H_{0}, H_{1}$, respectively.

$$
\begin{aligned}
& \beta_{0}=P\left\{K=0 \mid H_{0}\right\}=\left(R_{0}^{\prime}\right)^{N} \\
& \beta_{1}=P\left\{K=0 \mid H_{1}\right\}=\left(R_{1}^{\prime}\right)^{N}
\end{aligned}
$$

Here the detection probability $P_{d}$, the false alarm probability $P_{f}$ and the misdetection probability $P_{m}$ are given as follows:

$$
\begin{aligned}
P_{d}= & P\left\{H=1, K \leq 1 \mid H_{1}\right\} \\
& =1-\beta_{1} \\
P_{f}= & P\left\{H=1, K \geq 1 \mid H_{0}\right\}
\end{aligned}
$$

$$
=1-\beta_{0}
$$

and

$$
P_{m}=1-P_{d}
$$

From the above, it can be observed that the performance of cooperative sensing is not degraded due to imperfect reporting channel, the method auto-corrects the reporting error and thus create virtual perfect reporting for an imperfect channel

\section{SIMULATION RESULTS}

Simulation results are presented in this section to demonstrate the performance of cooperative spectrum sensing under auto correction reporting scheme and provide a comparison with the existing method, the results of the conventional method and the censoring method with quantization (with probability of fail sensing equal to 0.001) [9] are given for a comparison.

The simulation assumptions are as follows: the number of cognitive user is 10 users in the system, the average SNR between the primary user and any cognitive user is $10 \mathrm{~dB}$, and $P_{0}=0.5$.

Fig. (2) shows that in the proposed method the normalized average reporting bits has been decreased compared to the conventional method [9], and the two levels quantization method showing its efficiency. The censoring method creates a loss in the probability of false alarm due to the large 'No decision' region.

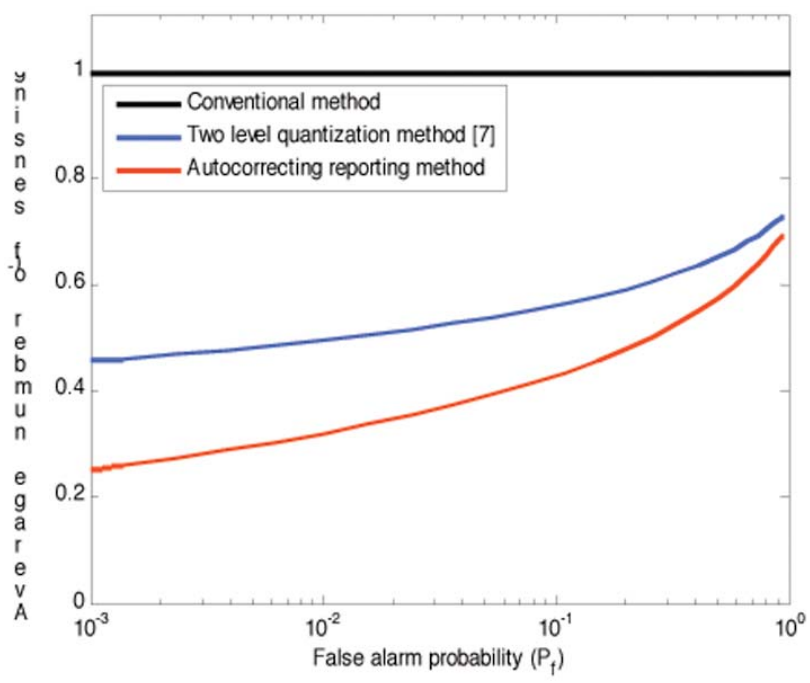

Fig. (2). The normalized average number of sensing bits $K^{\prime}$ vs. $P_{f}$, $N=10$, and $\mathrm{SNR}=10 \mathrm{~dB}$.

Fig. (3) illustrates the cooperative spectrum sensing performance of complementary receiver operating characteristic (ROC) $\left(p_{m} v s . p_{f}\right)$ of the, for the different reporting method.

The curves for the auto correction reporting scheme and conventional method (assuming perfect reporting channel for the conventional method) are the same which means there is 
no or unobserved performance loss for spectrum sensing reporting due to fading in reporting channel, which justify the analysis given in section III.

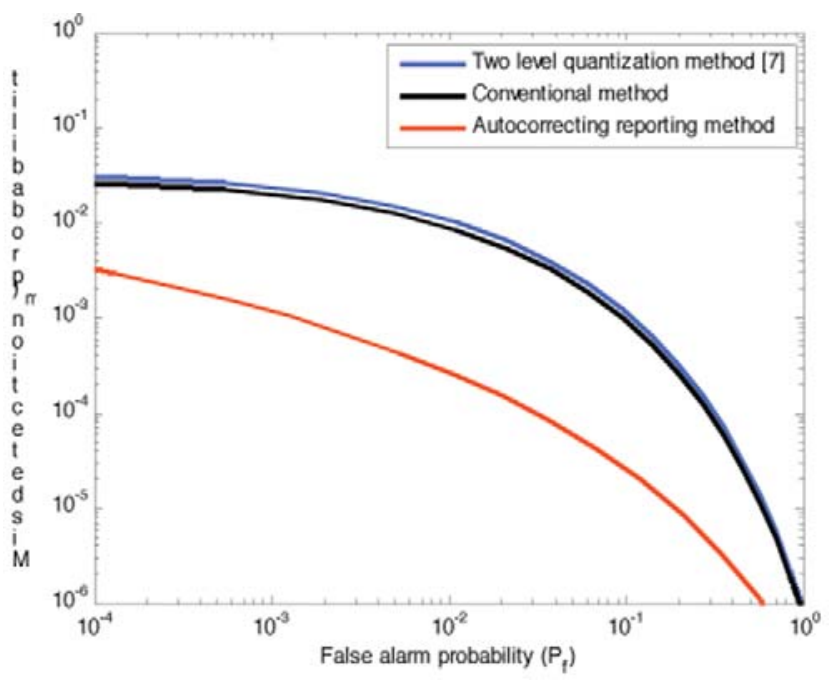

Fig. (3). Complementary Operating Characteristic (ROC) $\left(P_{m}\right.$ vs. $P_{f}$ ) of cooperative spectrum sensing , $N=10$, and $\mathrm{SNR}=10 \mathrm{~dB}$.

Fig. (4) shows the tradeoff between the spectrum sensing performance and the average number of reporting bits, i.e., $P_{M}$ vs. $\bar{K}$, for given false alarm probability, $P_{F}=0.0001$, $0.001,0.005$, respectively. It can be observed that, for a fixed false alarm probability, the missing probability $P_{m}$ changes a little when $K^{\prime}$ varies from 0.5 to 1 , which means that we can achieve a large reduction of number of sensing bits at a very little expense of performance loss.

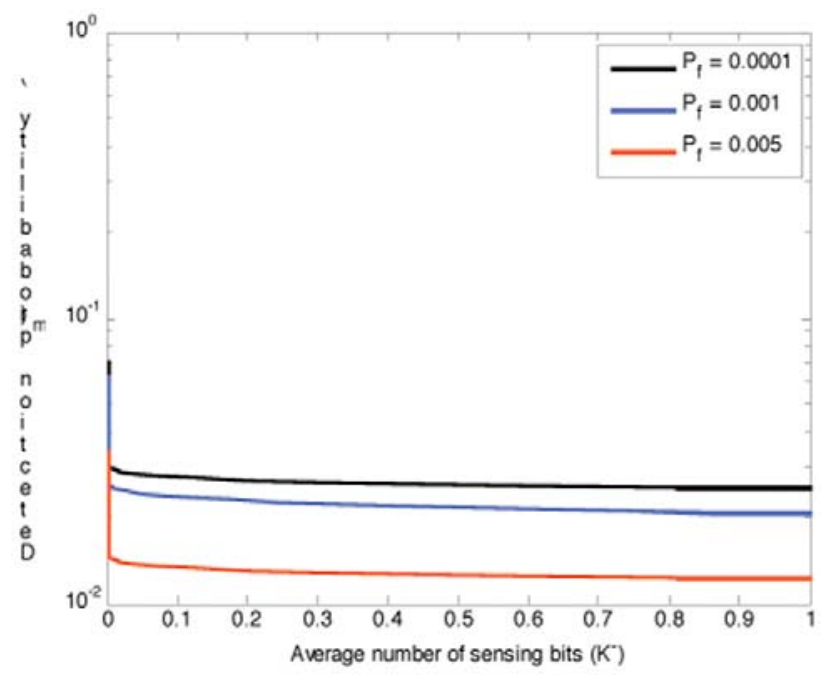

Fig. $\left(4 \overline{\overline{\bar{\gamma}}}_{\text {ss. } K}\right.$ ' for $P_{f}=0.0001,0.001,0.005, N=10$, SNR=10dB.

\section{CONCLUSIONS}

As far as the cognitive network grows, the coordination algorithm should have reduced protocol overhead. To decrease the average number of reporting bits for cooperative spectrum sensing in cognitive radio network a reporting method with auto error correction scheme is proposed here. It is shown that the method is reduced the average number of reported bits compared to the available existing method showing its superiority.

\section{ACKNOWLEDGMENT}

The first author gratefully acknowledges the support of this work by the Third World Organization for Women in Science (TWOWS), and Third World Academe of Science (TWAS).

\section{REFERENCES}

[1] Ghasemi and E. S. Sousa, "Collaborative spectrum sensing for opportunistic access in fading environments," in Proc. 1st IEEE Symp. New Frontiers in Dynamic Spectrum Access Networks, Baltimore, USA, Nov. 2005, pp. 131-136.

[2] D. Cabric, S. M. Mishra, and R. W. Brodersen, "Implementation issues in spectrum sensing for cognitive radios," in Proc. of Asilomar Conf. on Signals, Systems and Computers, Pacific Grove, CA, USA, Nov. 2004, pp. 772-776.

[3] G. Ganesan and Y. (G.) Li, "Cooperative Spectrum Sensing in Cognitive Radio - Part II: Multiuser networks," IEEE Trans. Wireless Commun., vol. 6, NO. 6, pp. 2214-2222, June 2007.

[4] J. N. Laneman and D. N. C. Tse, "Cooperative diversity in wireless networks: Efficient protocols and outage behaviour," IEEE Trans. Inform. Theory, vol. 50, Dec. 2004, pp. 3062-3080.

[5] G. Ganesan and Y. (G.) Li, "Cooperative Spectrum Sensing in Cognitive Radio - Part I: Two User networks," IEEE Trans. Wireless Commun., vol. 6, NO. 6, June 2007, pp. 2204-2213.

[6] D. Cabric, S. Mishra, D. Willkomm, R. Brodersen, and A. Wolisz, "A cognitive radio approach for usage of virtual unlicensed spectrum", in Proc. IST Mobile and Wireless Communications Summit, Dresden, Germany, June 2005.

[7] R. S. Blum, "Distributed detection for diversity reception of fading signals in noise," IEEE Trans. Inf. Theory, vol. 45, Jan. 1999, pp. 158-164.

[8] J. F. Chamberland and V. V. Veeravalli, "Decentralized detection in sensor networks," IEEE Trans. Signal Processing, vol. 51, Feb. 2003, pp. 407-416.

[9] Sun, W. Zhang, and K. B. Letaief, "Cooperative spectrum sensing for cognitive radios under bandwidth constraints," in Proc. IEEE International Wireless Communications and Networking Conference (WCNC 2007), Hong Kong, Marh 2007, pp. 1-5.

[10] C. Sun, W. Zhang, and K. B. Letaief "Cluster-Based Cooperative Spectrum Sensing in Cognitive Radio Systems" in Proc. IEEE International Conference on Communications (ICC 2007), Glasgow, Scotland, UK, June 2007, pp. 2511-2515.

[11] H. Urkowitz, "Energy detection of unknown deterministic signals," Proceedings of IEEE, vol. 55, April 1967, pp. 523-231.

[12] I. S. Gradshteyn and I. M. Ryzhik, Table of Integrals, Series, and Products, 5th ed. Academic Press, 1994. 